\title{
On the performance of a viscoelastic constitutive model for micro injection moulding simulations
}

\author{
A. Gava, G. Lucchetta* \\ University of Padova, Department of Innovation in Mechanics and Management, Via Venezia, 1 - 35131 Padova, Italy
}

Received 16 September, 2011; accepted in revised form 5 December 2011

\begin{abstract}
The numerical simulation of the injection moulding process involving microstructures presents several challenges, mainly due to the surface effects that dominate the flow behaviour at the microscale. In this paper a new approach, which employs weld lines as flow markers, is used to evaluate whether the numerical codes that are normally used to simulate the conventional injection moulding process, are suitable to characterize the melt flow patterns in the filling of micro features. The Cross-WLF viscous model and the Giesekus viscoelastic model were evaluated using 3D models of a micro part implemented in two different numerical codes. A micro cavity was designed in order to compare the results of numerical simulations and experiments. While the viscous simulations were found to be inappropriate for multi-scale structures, the accuracy of micro filling predictions was significantly improved by implementing a viscoelastic material model.
\end{abstract}

Keywords: rheology, modelling and simulation, micro injection moulding

\section{Introduction}

Due to their cost effectiveness and rapid response, numerical simulations have become an increasingly important tool for the evaluation of part design, mould geometry and processing parameters in injection moulding. Numerical investigations are able to estimate aspects of the physical model which otherwise would be difficult to quantify. They allow quick responses on what will be the effects of process parameters changes on the final part. Although Computer Aided Engineering (CAE) has been used with increasing success in the design and manufacture of polymer products and processes, the simulation of the injection moulding process involving microstructures $(\mu \mathrm{IM})$ presents many challenges. The flow behaviour of polymer melts in mould micro cavities is not fully understood. It is believed that, due to the large surface-to-volume ratio, surface effects dominate the flow behaviour at the microscale [1]. Kemann et al. [2] showed that stan- dard injection moulding simulation packages are not able to describe all of the effects in micromoulding. The rheological data used in current packages are obtained from macroscopic experiments and they are not suitable for modelling microscale flows. In fact, when flowing in micro channels a polymer has a strong tendency to slip [3]. If a classic no-slip boundary condition is used in current packages, the consequences of wall slip cannot be predicted. Furthermore, the microscale dimensions of features and rapid filling rates typically occurring within micro cavities, ensure that the values of shear rate, experienced by the polymer during the micro moulding process, are orders of magnitude higher than those experienced in conventional injection moulding. The extensional behaviour in contraction flow or the pressure influence on the viscosity cannot be neglected as well. Also, the mould/ melt heat transfer coefficient was found to be a critical factor in determining the filling lengths [4]. A

\footnotetext{
${ }^{*}$ Corresponding author, e-mail: giovanni.lucchetta@unipd.it (c) BME-PT
} 
significant decrease in the Nusselt number was observed concerning the laminar flow in microscale channels. This means that a constant heat transfer coefficient may not be applicable to the heat transfer simulation involving flow through microscale channels.

The assumption of a generalized Newtonian fluid is generally used for traditional injection moulding because the importance of elasticity compared to viscous effects is negligible. Because of high deformation rates during the injection phase of high speed micro injection moulding, it is expected that elastic effects will occur. Therefore there is a need to translate the complex rheological behaviour of polymeric fluids into suitable equations, and to use these models to predict flow in micro cavities. During the last decade substantial progress has been made in the numerical simulation of viscoelastic flows. Extensions to non-isothermal and three-dimensional viscoelastic simulations are in progress [5]. Several constitutive equations have been proposed, but none of them has been proven to be superior to others [6].

The main objective of this work is to evaluate whether the present numerical commercial codes are suitable to characterize melt flow patterns in a micro-cavity, using both Autodesk Moldflow Insight ${ }^{\circledR}$, i.e. a dedicated simulation software, and a general purpose fluid dynamic finite element (FEM) code such as Ansys Polyflow ${ }^{\circledR}$. This paper also reports on the suitability of $3 \mathrm{D}$ general purpose computational fluid dynamic (CFD) software to be used for injection moulding simulations.
The approach proposed to compare the predictions of numerical simulations to moulding results (Figure 1) consists in the determination of the flow pattern by using weld lines as flow markers. This is an alternative technique to the 'short shots' method, which predicts the shape of the free surfaces with a large approximation due to stress relaxation and thermal contraction.

\section{Experimental setup and data acquisition}

The mould micro cavity considered in this study was designed to create an effective response variable to compare the results of numerical simulations and experiments. Obstacles as high as the total depth of the cavity were created. In this way, the melt flow was not allowed to climb over the features and when the separated melt fronts rejoined, at some downstream location, weld lines were formed. Geometries and dimensions (Figure 2) were selected according to existing industrial devices (blood separators and micro pumps) and in order to exalt such factors that change their relevance when shifting from conventional to micro injection moulding, such as:

- elongational flow,

- heat transfer in different thickness,

- wall slip,

- elastic behaviour of polymers.

In the first part of the cavity, a convergent geometry was created in order to pull out the extensional flow and elastic behaviour of the melt in convergent/ divergent geometries. A step was created in the middle part of the cavity in order to originate a three

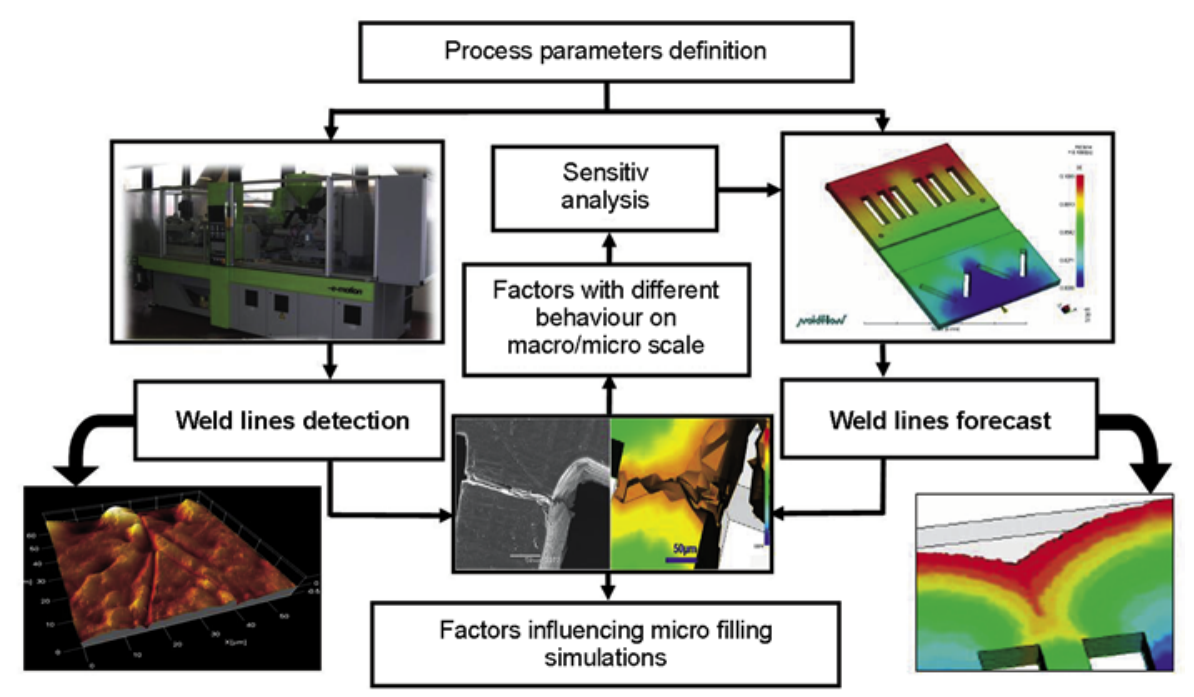

Figure 1. Proposed approach for the filling validation 


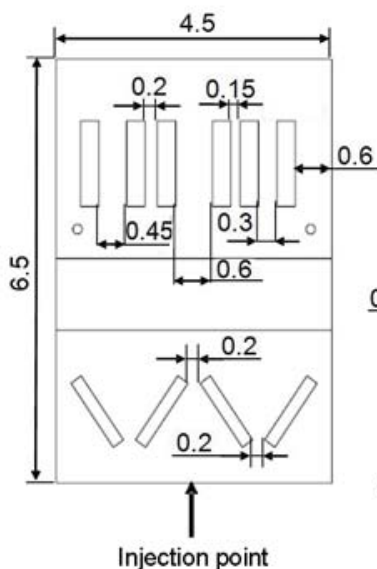

a)

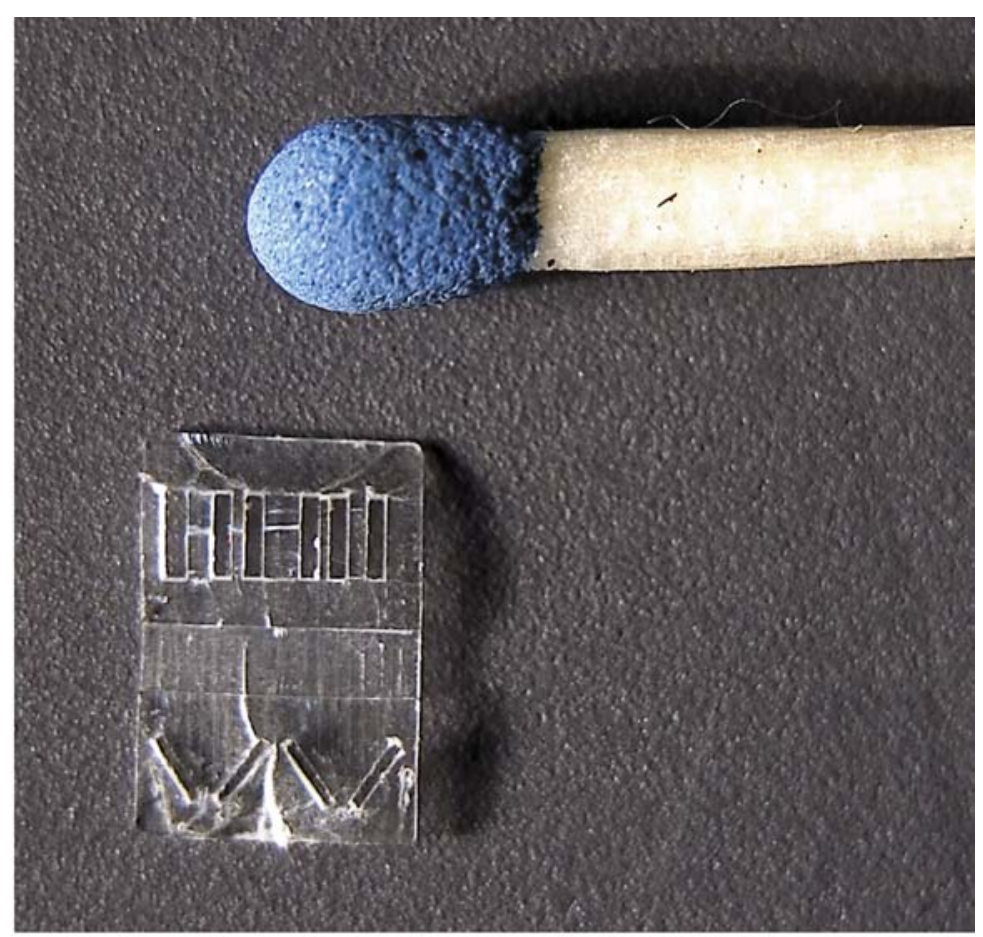

b)

Figure 2. Micro part design (part volume $6 \mathrm{~mm}^{3}$ ) (a) and manufactured part (b)

dimensional flow and to align the flow front before the micro channels entrance; furthermore, the direction of the expected weld line, after the two holes, could give information regarding the shape of the flow front in the second part of the cavity.

Channels as wide as 150, 200, 300, 450 and $600 \mu \mathrm{m}$ were created in the second part. Heat transfer in variable thickness and different rheological models were considered. The gate $(400 \mu \mathrm{m}$ wide and $250 \mu \mathrm{m}$ thick) was realised as small as possible, in order to approximate it as an injection point.

The experiments were performed using a high fluidity class polystyrene, the PS 143 E produced by BASF (Ludwigshafen, Germany). Polystyrene is relevant in micro injection moulding for its very low viscosity, good biocompatibility, high optical clarity, high transparency and high impact strength compared to silicon or glass. The polymer was injected into the mould cavity setting a constant speed profile of $350 \mathrm{~mm} / \mathrm{s}$. The melt temperature in the feeding zone was maintained at $230^{\circ} \mathrm{C}$. The mould temperature was controlled by a heater and maintained at $70^{\circ} \mathrm{C}$.

In order to obtain a closer control on the boundary conditions and a good comparison between simulations and experiments, temperature and pressure transducers were positioned near the cavity surface.
The following parameters were acquired during the injection moulding process:

- hydraulic pressure,

- ram position,

- cavity pressure near gate,

- cavity surface temperature.

The pressure and temperature transducers were mounted in the fixed mould part.

In order to analyse the filling of the micro cavity, the position and direction of weld lines were used as measurable outputs. To describe the shape of the weld lines, an accurate measurement of the weld line path on the sample was required. The investigation strategy consisted in detecting the $X$ and $Y$ coordinates of points on the weld lines with an optical coordinate measurement machine (CMM) (Video Check IP 400, Werth Messtechnik, Giessen, Germany) and to plot them in a determined and repeatable reference system.

Measuring uncertainty was estimated taking into account the optical CMM calibration on the measured dimensional range, repeatability of the measurement on 5 repetitions, CMM resolution, definition of the measurand and the influence of the temperature (i.e. expansion of the plastic due to temperature variations). The combined expanded measuring uncertainty, calculated with a coverage factor $k=2$ 


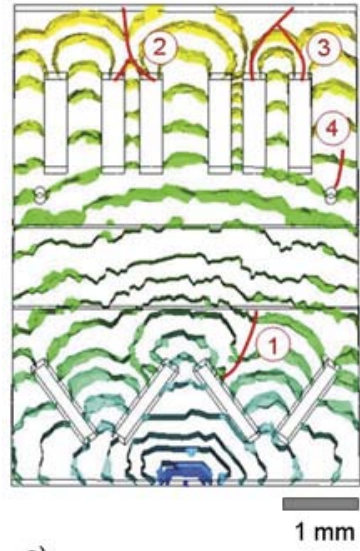

a)

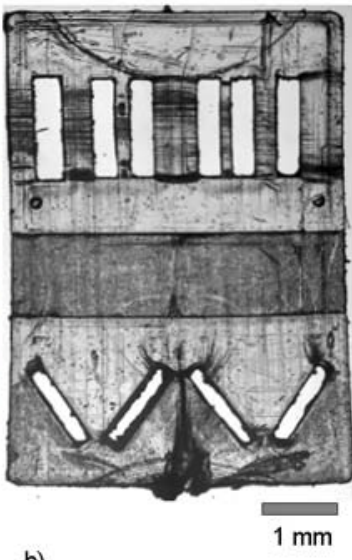

b)

Figure 3. Moldflow ${ }^{\circledR}$ filling simulation results (a) and injection moulded part (b)

(level of confidence 95\%), resulted in $4 \mu \mathrm{m}$ on the $X$ direction and in $3 \mu \mathrm{m}$ on the $Y$ direction. The investigated area and the injection moulded part are represented in Figure 3 [7].

\section{Viscous and viscoelastic material characterization}

In order to have a closer control on the boundary conditions setup and to improve numerical simulation results, the PS 143 E by BASF was characterized both by means of a rotational and a capillary rheometer.

Steady shear measurements were performed on a dual bore capillary rheometer (Rheo 2500, Ceast, Pianezza, Italy) at three different temperatures $\left(190,210\right.$ and $\left.230^{\circ} \mathrm{C}\right)$ with a shear rate range of 100 to $10000 \mathrm{~s}^{-1}$. The shear-dependent viscosity data were fitted to the Cross model (Figure 4, Equation (1)):

$$
\eta_{\mathrm{s}}=\frac{\eta_{0}}{1+\left(\frac{\eta_{0} \dot{\gamma}}{\tau^{\star}}\right)^{1-\mathrm{n}}}
$$

where $\eta_{0}$ is the viscosity at zero shear and $\tau^{\star}$ and $n$ are data-fitted coefficients. The effect of temperature on viscosity was accounted by means of the Williams-Landel-Ferry (WLF) model (as defined by Equations (2)-(4)):

$$
\begin{aligned}
& \eta_{0}=D_{1} \exp \left[\frac{-A_{1}\left(T-T^{\star}\right)}{A_{2}+\left(T-T^{\star}\right)}\right] \\
& T^{\star}=D_{2}+D_{3} \cdot P \\
& A_{2}=\widetilde{A}_{2}+D_{3} \cdot P
\end{aligned}
$$

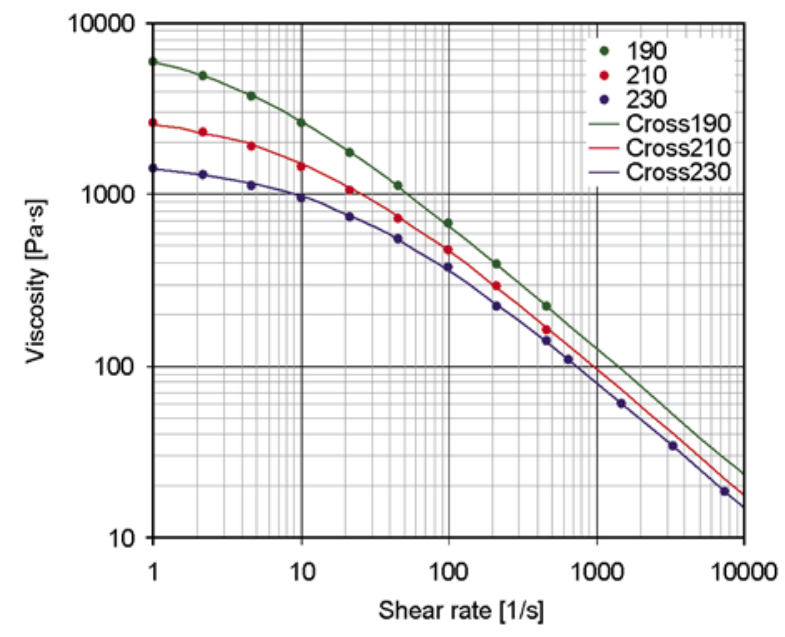

Figure 4. Experimental data and Cross-WLF fitting for the steady shear viscosity of PS 143E

Table 1. Cross-WLF model constants for PS 143E

\begin{tabular}{|l|c|l|c|l|c|}
\hline \multicolumn{2}{|c|}{ Cross } & \multicolumn{4}{c|}{ WLF } \\
\hline$n$ & 0.25433 & $D_{1}[\mathrm{~Pa} \cdot \mathrm{s}]$ & $9.47833 \cdot 10^{26}$ & $A_{1}$ & 56.342 \\
\hline$\eta_{0}[\mathrm{~Pa} \cdot \mathrm{s}]$ & 1548.89 & $D_{2}[\mathrm{~K}]$ & 428.15 & $\widetilde{A}_{2}[\mathrm{~K}]$ & 2.192 \\
\hline$\tau^{\star}[\mathrm{Pa}]$ & 31210.18 & $D_{3}[\mathrm{~K} / \mathrm{Pa}]$ & $2.1 \cdot 10^{-7}$ & & \\
\hline
\end{tabular}

where $D_{1}, D_{2}, D_{3}, A_{1}$ and $\widetilde{A}_{2}$ are constants to be determined while $T^{\star}$ is a reference temperature [8]. Model constants implemented in the numerical simulations are summarized in Table 1. Pressure dependence of viscosity was measured by using a throttle apparatus arranged in series with the die of the capillary rheometer [8]. Data obtained from experiments in a pressure range of $40 \mathrm{MPa}$ were fitted according to the Cross model and the $D_{3}$ coefficient was estimated as $2.1 \cdot 10^{-7} \mathrm{~K} / \mathrm{Pa}$.

Due to the high deformation rates, extensional deformations also play a significant role in the process. Thereby, for the viscous simulation the Moldflow $^{\circledR}$ 'unified' viscosity model for mixed shear and extension deformations was used. In this model the apparent viscosity is modelled as a function of the extension rate and the shear viscosity using the extension viscosity model coefficients. These coefficients were determined using experimental pressure measurements in convergent flow. The apparent viscosity, $\eta_{\mathrm{a}}$, is modelled according to Equation (5):

$\eta_{\mathrm{a}}(T, P, \dot{\gamma}, \dot{\varepsilon})=f(\dot{\varepsilon}) \eta_{\mathrm{s}}(T, P, \dot{\gamma})$

where $\eta_{\mathrm{s}}$ is the shear viscosity calculated by the Cross-WLF model and $f$ is a transition function defined by Equation (6): 
$f(\dot{\varepsilon})=1+\frac{A \cdot \dot{\varepsilon}}{B+\dot{\varepsilon}}$

where the elongational rate is determined from the entrance pressure loss in a capillary according to the Cogswell approach [9], while $A$ and $B$ are datafitted coefficients respectively related to the magnitude of the elongational effect and to the extension rate of the transition to strong elongational stresses (Table 2).

On the other hand, for the viscoelastic simulation dynamic experiments were performed on a rotational rheometer (ARES, TA Instruments, New Castle, USA) at three different temperatures $(190,210$ and $230^{\circ} \mathrm{C}$ ) in a frequency range $\omega=0.1-100 \mathrm{~s}^{-1}$. A parallel disk geometry was chosen rather than a cone plate apparatus for practical reasons. The samples, prepared by compression moulding of the pellets, were maintained under nitrogen atmosphere to avoid polymer degradation by oxidation. Measurements were performed in frequency sweep mode with a 5\% deformation amplitude. Three measurement points per decade were taken at increasing frequencies. The rheological functions were shifted to a master-curve at reference temperature of $230^{\circ} \mathrm{C}$ using the time temperature superposition [10]. The horizontal shift factor $a_{\mathrm{T}}\left(T, T_{\text {ref }}\right)$ follows from the loss angle $(\delta)$ and the vertical shift factor $b_{\mathrm{T}}\left(T, T_{\text {ref }}\right)$ from the dynamic modulus $\left(G_{\mathrm{d}}\right)$ (see Equations (7) and (8)):

$\delta(\omega, T)=\delta\left(\omega_{\mathrm{a}}, T_{\mathrm{ref}}\right)$

$G_{\mathrm{d}}(\omega, T)=b_{\mathrm{T}} G_{\mathrm{d}}\left(\omega_{\mathrm{a}_{\mathrm{T}}}, T_{\mathrm{ref}}\right)$

The shift factors obtained experimentally (Table 3) were plotted as a function of temperature and fitted according to the WLF equation.

Dynamic experiments allowed to determine the relaxation spectrum, i.e. the set of relaxation times,

Table 2. Extension viscosity coefficients for PS 143E

\begin{tabular}{|c|c|c|}
\hline Coefficients & A & B \\
\hline & 0.309 & 297.26 \\
\hline
\end{tabular}

Table 3. Horizontal and vertical shift factors obtained from experimental data

\begin{tabular}{|c|l|l|}
\hline Temperature $\left[{ }^{\circ} \mathbf{C}\right]$ & \multicolumn{1}{|c|}{$\mathbf{a}_{\mathbf{T}}$} & $\mathbf{b}_{\mathbf{T}}$ \\
\hline 190 & 8.62998 & 1.25456 \\
\hline 210 & 2.70511 & 1.21066 \\
\hline 230 & 1 & 1 \\
\hline
\end{tabular}

by fitting with experimental data the expressions predicted by the viscoelastic Giesekus model in the case of small-amplitude oscillatory shear flow. The Giesekus model was selected because it gives satisfactory predictions in the standard rheometrical tests that appear most relevant for the flow under consideration [11]. The Giesekus model, belonging to the class of Maxwell-type differential constitutive equations, is capable of describing the complex rheological behaviour of a material at various deformation histories. By introducing several uncoupled or coupled discrete relaxation modes it can be shown that the area of concordance between predictions of the model and experimental results for polymer solutions can be considerably extended [12]. The model can be written as shown by Equation (9):

$$
\lambda \stackrel{\overline{\bar{\tau}}}{\underline{\bar{\tau}}}+\left(\overline{\bar{I}}+\frac{\alpha \lambda}{\eta_{\mathrm{v}}} \overline{\bar{\tau}}_{\mathrm{v}}\right) \overline{\bar{\tau}}_{\mathrm{v}}=2 \eta_{\mathrm{v}} \overline{\bar{D}}(\overline{\bar{u}})
$$

where $\lambda$ is the relaxation time, $\overline{\bar{\tau}}_{\mathrm{v}}$ is the viscoelastic part of the extra-tensor, $\eta_{\mathrm{v}}$ is the viscoelastic contribution to viscosity, $\alpha$ is a dimensionless mobility parameter and $\bar{D}$ is the rate of strain tensor [11]. Multiple relaxation times have been used in order to better fit the viscoelastic behaviour at different shear rates and improve accuracy of simulation results. Complex viscosity, loss and storage moduli were fitted according to a 4-mode Giesekus model (Figure 5).

In the model, nonlinear effects are introduced by taking into account an average anisotropy of the molecular conformation during flow. The strength of influence to the conformation and the retroaction

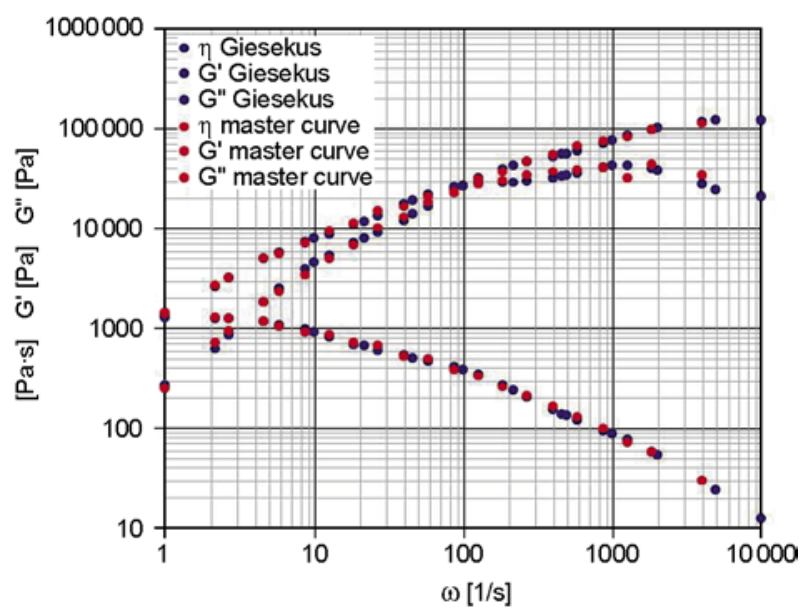

Figure 5. Comparison of complex viscosity, loss and storage moduli predicted by a 4-mode Giesekus model with experimental data for PS at reference $T=$ $230^{\circ} \mathrm{C}$ 


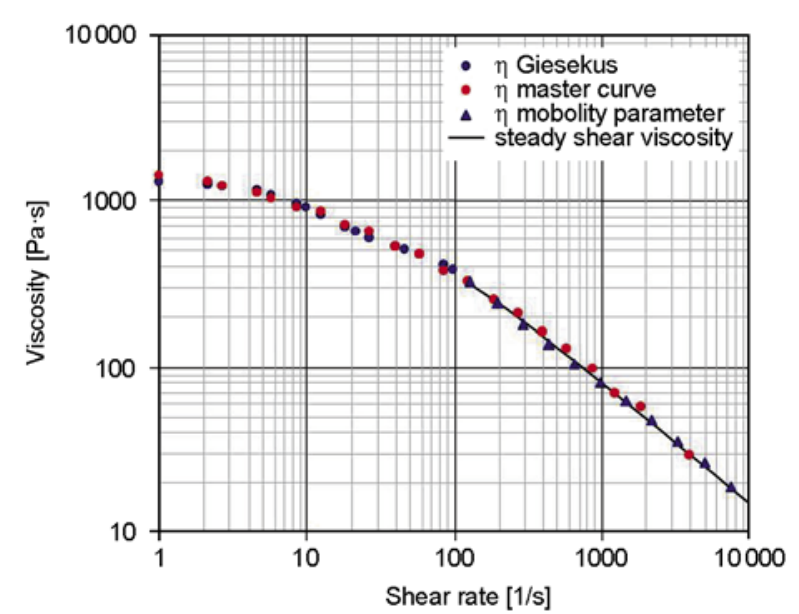

Figure 6. Fitting of non-linear parameter. Comparison of steady shear viscosity (as a function of shear rate) with linear viscosity (as a function of frequency).

to the flow is determined by an anisotropic mobility parameter $\alpha$. At $\alpha=0$ the isotropic Maxwell model is recovered. When $\alpha$ is set to unity the model predicts similar behaviour in elongation flows as the corotational Maxwell model. At intermediate values of $\alpha$, the Giesekus model fits steady and transient shear flows better than any other differential constitutive equation [5]. A best fit for the $\alpha$ parameter (Figure 6) was obtained by minimizing the deviation from the steady shear experiments conducted on the capillary rheometer with the function defined by Equation (10):

$R=\sum_{\mathrm{j}=1}^{\mathrm{N}}\left[\left(\frac{G^{\prime}\left(\omega_{\mathrm{j}}\right)}{G^{\prime}{ }_{\text {exp } \mathrm{j}}}-1\right)^{2}+\left(\frac{G^{\prime \prime}\left(\omega_{\mathrm{j}}\right)}{G^{\prime \prime}{ }_{\text {exp.j }}}-1\right)^{2}\right]$

where $j$ indicates the individual data points.

The knowledge of both the flow field and the fluid properties determines the character of the flow. In particular, the Deborah number is used to characterize the fluid elasticity, which is defined as the ratio of the material characteristic relaxation time $\lambda$ to the characteristic flow time $t$ (Equation (11)):

$D e=\frac{\lambda}{t}$

The weighted relaxation time was calculated as shown by Equation (12):

$$
\lambda=\frac{\sum_{i=1}^{4} \eta_{\mathrm{i}} \lambda_{\mathrm{i}}}{\sum_{\mathrm{i}=1}^{4} \eta_{\mathrm{i}}}
$$

The estimated Deborah number is 11.6. This value confirms the hypothesis that the filling behaviour is influenced by viscoelastic effects. In this case the value calculated for the Deborah number is not comparable to values typical of the conventional injection moulding process. This establishes that the elastic behaviour of the polymer melt has to be considered.

\section{Numerical simulations}

As a first approach a three-dimensional finite element Moldflow ${ }^{\circledR}$ analysis was performed in order to simulate the micro injection moulding process. The main material functions considered are the 'unified' viscosity model and the two-domain Tait model for the $p v T$ data. Non-Newtonian, non-isothermal flow solutions were obtained by solving the momentum, mass and energy governing equations. No-slip boundary conditions were imposed on the cavity walls filled by the polymer, while on the unfilled part, a free boundary condition allowed for the formation of the typical fountain flow. The melt temperature is a potential problematic parameter in terms of modelling. In practice, the melt temperature is only indirectly controlled through the barrel temperature zones. It was therefore decided to consider it as equal to the barrel temperature. The mould temperature was defined as the mean value of the mould surface acquired by the temperature transducers. The simulations were performed implementing the ram speed profile as set in the machine. The numerical simulation was carried out using the 3D mesh shown in Figure 7. A sensitivity analysis of software simulation to the mesh dimension was conducted. It was decided, as a result, to mesh the

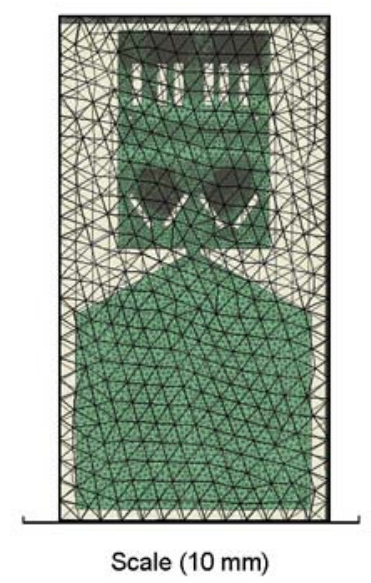

Figure 7. Mesh of the 3D model considered in Moldflow ${ }^{\circledR}$ simulation 
part model together with the feeding system and to decrease the mesh size in the cavity zones where the weld lines were experimentally detected.

As a second approach a three dimensional viscoelastic simulation was performed in the Ansys Polyflow ${ }^{\circledR}$ environment. Polyflow ${ }^{\circledR}$ is a finite-element program primarily designed for the analysis of industrial flow processes dominated by non-linear viscous phenomena and viscoelastic effects. The theoretical foundation is provided by the general principles of continuum mechanics, together with phenomenological and kinetic theoretical models for describing the rheological behaviour of the fluid.

The model was created and meshed in Gambit ${ }^{\circledR}$ and simulations were performed in Polydata ${ }^{\circledR}$ implementing the same previous boundary conditions. Stress (one stress field for each mode, or relaxation time), velocity and pressure are computed simultaneously. Furthermore, the problem involves flow, heat transfer by conduction and convection and heat generation by viscous dissipation. Energy, momentum and mass governing equations were solved in the fluid domain implementing the viscoelastic properties of the material.

In the viscoelastic simulation, the total extra-stress tensor is divided into a purely viscous part (Newtonian) and a viscoelastic part (Equation (13)):

$\overline{\bar{\tau}}=\overline{\bar{\tau}}_{\mathrm{n}}+\overline{\bar{\tau}}_{\mathrm{v}}$

where the subscripts $n$ and $v$ stand for Newtonian and viscoelastic contributions, respectively. The Newtonian part, which can be seen as the stress response associated with fast relaxation modes, is written as shown by Equation (14):

$\overline{\bar{\tau}}_{\mathrm{v}}=2 \eta_{\mathrm{n}} \overline{\bar{D}}(\overline{\bar{u}})$

$\overline{\bar{\tau}}_{\mathrm{v}}$ is computed according to the Giesekus model [11].

When a multi-mode viscoelastic model is used, the total extra-stress tensor is decomposed into a sum of individual viscoelastic components and any purely-viscous component. To prevent ambiguous definition of purely-viscous component, the corresponding viscosity factor is defined together with the first mode. Consequently, the remaining modes will not contain any purely-viscous components.

In this work a numerical analysis was performed defining three solid and one fluid sub-domains (Figure 8):

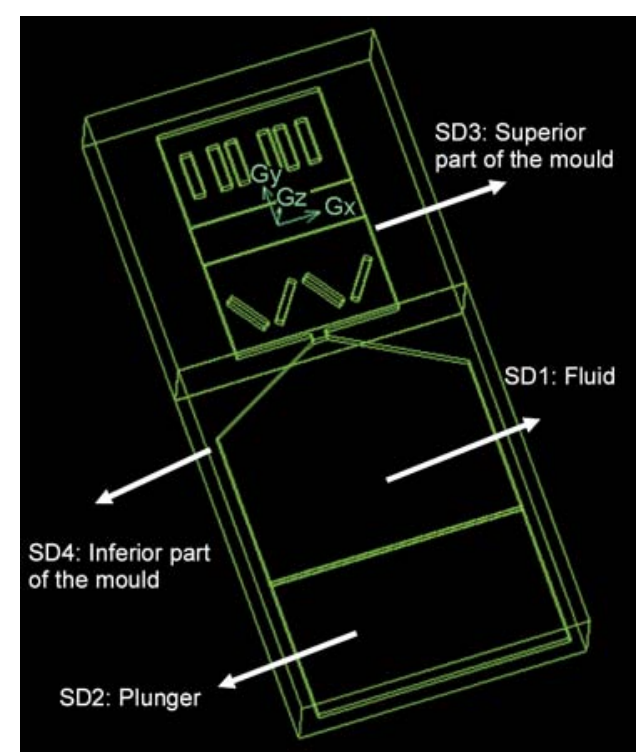

Figure 8. Sub-domains definition defined on Polyflow ${ }^{\circledR}$ environment

- a fluid domain (SD1)

- a plunger (SD2)

- the inferior part of the mould (SD3)

- the superior part of the mould (SD4).

The finite element meshes are represented in Figure 9. Three dimensional finite elements are defined for fluid gob, mould and plunger. In particular the fluid domain and the superior part of the mould are meshed by triangles while the inferior part of the mould and the plunger are meshed by quadrilaterals. The 3D mesh model is represented in the initial configuration, before pressing the fluid. At a prescribed time the plunger moves downwards and presses this fluid domain in the mould cavity.

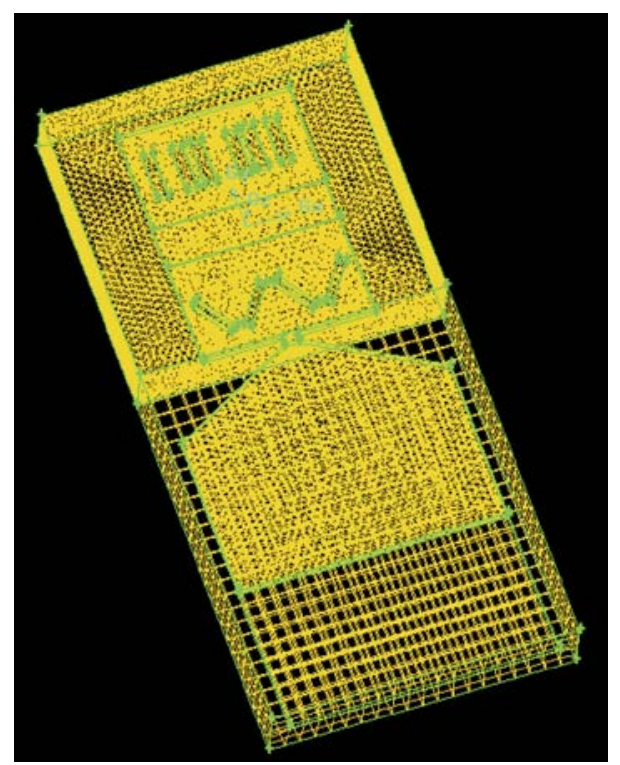

Figure 9. Finite element mesh of the model in Polyflow ${ }^{\circledR}$ environment 
A major difficulty to be overcome in the simulation arises from the fact that the filling domain evolves considerably over time. The position of the front is an unknown, which means that the limit of the domain under investigation (i.e. filled with polymer melt) is an unknown. This is called a free surface problem and has given rise to a large number of methods, which can be classified into two categories. The first approach consists in using control volumes defined on a fixed mesh, covering the entire domain to be filled, with the use of an additional variable representing the volume fraction of the injected polymer within the control volumes (and without front discretization). The second approach is based on accurately tracking the flow front and adapting the mesh, at each time step, in order to cover only the filled domain. This latter front tacking-remeshing approach was selected to solve the micro injection moulding problem, since an accurate representation of the front at an affordable computational cost was required in this small scale problem. Indeed, the second class of methods requires refined finite element meshes in the front vicinity. Besides this, the exact position of front meetings was intrinsically interesting as related to the weld line position. The fluid domain was, therefore, considered as a free surface and a Lagrangian remeshing technique was applied. In the Polyflow ${ }^{\circledR}$ environment, remeshing techniques are based only on the positions and displacements of the boundary nodes, and not on kinematic considerations, unless a Lagrangian or streamwise method is used for remeshing. Tangential remeshing preserves the original node distribution along a surface for three dimen-

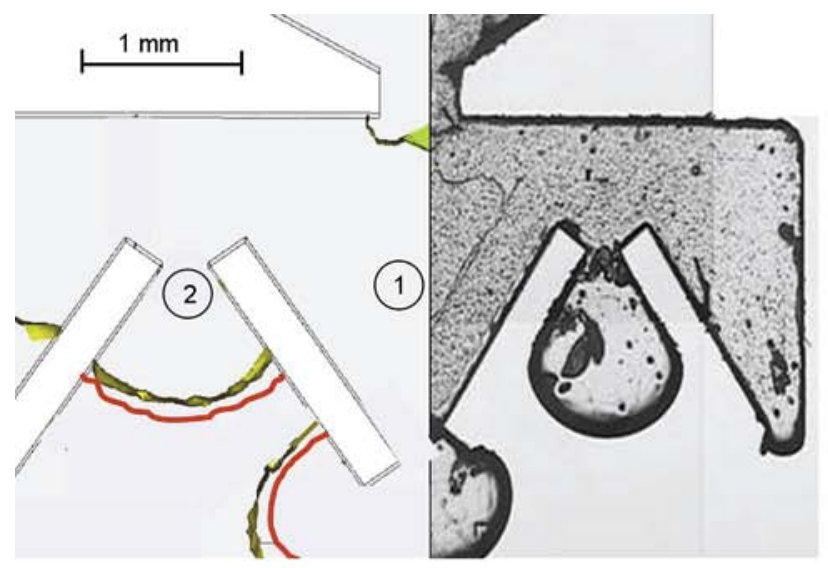

a) sional moving domains. In transient iterative parameters a maximum value of $10^{-6} \mathrm{~s}$ was set as the time step in order to contain deformation of the elements before remeshing.

\section{Results}

An extensive measurement campaign of the experimental and simulated weld lines positions was carried out covering weld lines located in several areas of the micro component. Two different outputs were considered: the weld line number 1 and the line 1 of the weld line number 3 . These two weld lines were selected because they originate in zones of the micro cavity where the elastic behaviour of the fluid should not be neglected. In correspondence with the first part of the micro cavity, a contraction flow is opposed to an expansion flow. Two different streamlines were acquired at $1.2 \mathrm{~ms}$ both in Moldflow ${ }^{\circledR}$ (yellow) and in Polyflow ${ }^{\circledR}$ (red) simulations (Figure 10). In the expansion flow, the streamline in the viscoelastic simulation reveals a more elastic behaviour if compared to the viscous simulation. On the other hand, the streamline at the contraction exit is delayed due to normal stresses and resistance to elongational deformation during the contraction flow. As a consequence, the viscoelastic numerical weld line moves towards the experimental one. As a second case, the horizontal line in the $150 \mu \mathrm{m}$ wide micro channel was considered. Figure 11 shows how the viscous numerical simulation overpredicts the ease at which the polymer would flow through this channel; on the other hand, the viscoelastic one underpredicts (with a lower absolute error) the weld line position. The dif-

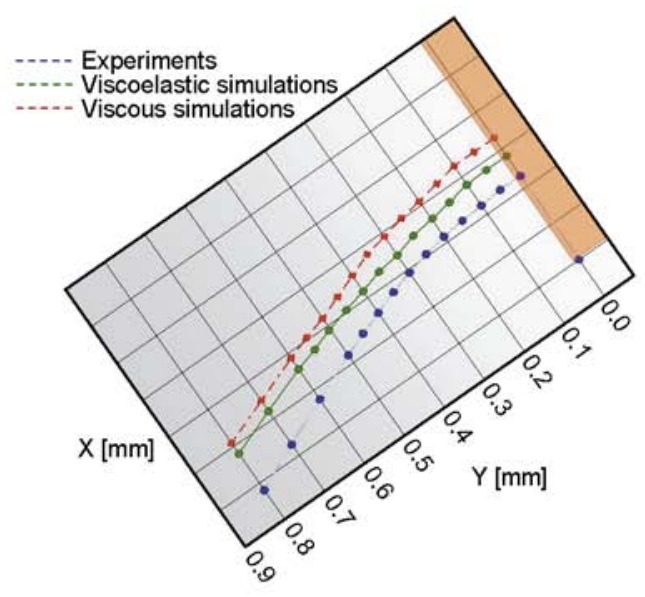

b)

Figure 10. Streamlines at $1.2 \mathrm{~ms}$ (a) and subsequent weld line formation (b) 


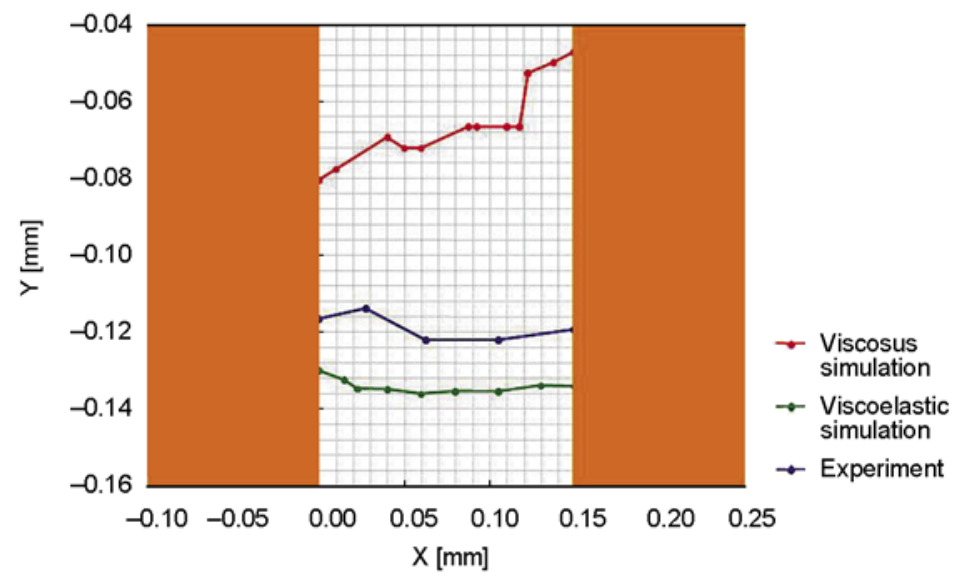

Figure 11. Line 1 of the weld line number 3

ference may be justified considering geometrical constraints and the viscoelastic nature of the polymer itself. Normal stresses and resistance to elongational deformation reduce the filling length in the micro channel. This effect is not as clear as in the other channels with higher dimensions, because as the channel dimensions decrease the material appears to be more rigid due to the constraints at the wall.

Differences between numerical and experimental results may be related to inadequate boundary conditions. No-slip conditions were imposed on the cavity walls filled by the polymer whereas wall slip is expected to occur due to the increased shear stress. A complete validation of the approach will be possible when more reliable models about local viscosity and heat transfer will be available.

\section{Conclusions}

In this paper a new approach, which employs weld lines as flow markers, is used to evaluate whether the commercially available numerical codes are suitable to characterize the melt flow patterns in the micro moulding process. A micro cavity was designed and manufactured in order to create an effective response variable to compare the results of numerical simulations and experiments. Conventional three dimensional simulations were tested and found to be inappropriate for multi-scale structures, typically in micro-injection moulded parts. It was expected that differences between experiments and numerical investigations would be due to the assumption of a generalized Newtonian fluid, generally used for traditional injection moulding, where the importance of the material elasticity compared to viscous effects appears to be negligible. Because of high deformation rates during the injection phase, it was expected that viscoelastic effects might occur.

Careful material characterization was conducted by means of both capillary and rotational rheometry and data obtained were fitted according to a non linear viscoelastic model (Giesekus model). Three dimensional viscoelastic numerical simulations were then performed to evaluate whether the implementation of a viscoelastic material model could improve the accuracy of micro filling simulations. Improvements in the viscoelastic simulation results were observed in predicting the weld lines position. Further differences between experiments and numerical simulations are to be related to the absence of a robust local heat transfer model.

\section{References}

[1] Ho C-M., Tay Y-C.: Micro-electro-mechanical-systems (MEMS) and fluid flows. Annual Review of Fluid Mechanics, 30, 579-612 (1998). DOI: 10.1146/annurev.fluid.30.1.579

[2] Kemmann O., Weber L., Jeggy C., Magotte O., Dupret F.: Simulation of the micro injection molding process. in 'Proceedings of SPE Annual Technical Conference - ANTEC 2000. Orlando, Florida, USA' 576-580 (2000).

[3] Chien R-D., Jong W-R., Chen S-C.: Study on rheological behavior of polymer melt flowing through microchannels considering the wall-slip effect. Journal of Micromechanics and Microengineering, 15, 13891396 (2005). DOI: $\underline{10.1088 / 0960-1317 / 15 / 8 / 003}$ 
[4] Yu L., Koh C. G., Lee L. J., Koelling K. W., Madou M. $\mathrm{J}$. : Experimental investigation and numerical simulation of injection molding with micro-features. Polymer Engineering and Science, 42, 871-888 (2002). DOI: $10.1002 /$ pen.10998

[5] Peters G. W. M., Schoonen J. F. M., Baaijens F. P. T., Meijer H. E. H.: On the performance of enhanced constitutive models for polymer melts in a cross-slot flow. Journal of Non-Newtonian Fluid Mechanics, 82, 387 427 (1999).

DOI: 10.1016/S0377-0257(98)00173-6

[6] Larson R. G.: A critical comparison of constitutive equations for polymer melts. Journal of Non-Newtonian Fluid Mechanics, 23, 249-269 (1987).

DOI: $10.1016 / 0377-0257(87) 80021-6$

[7] Gava A., Tosello G., Hansen H. N., Salvador M., Lucchetta G.: A new approach for the validation of filling simulations in micro injection moulding. in 'Proceedings of the $9^{\text {th }}$ International Conference on Numerical Methods in Industrial Forming Processes - NUMIFORM, Porto, Portugal' 307-312 (2007).

DOI: $10.1063 / 1.2740829$
[8] Cardinaels R., Van Puyvelde P., Moldenaers P.: Evaluation and comparison of routes to obtain pressure coefficients from high-pressure capillary rheometry data. Rheologica Acta, 46, 495-505 (2007).

DOI: $10.1007 / \mathrm{s} 00397-006-0148-5$

[9] Macosko C. W.: Rheology: Principles, measurements and applications. Wiley-VCH, New York (1994).

[10] Ferry J. D.: Viscoelastic properties of polymers. Wiley, New York (1980).

[11] Giesekus H.: A simple constitutive equation for polymer fluids based on the concept of deformation-dependent tensorial mobility. Journal of Non-Newtonian Fluid Mechanics, 11, 69-109 (1982).

[12] Armstrong R. C., Brown R. A., Quinzani L. M., McKinley G. H., Byars J. A.: Measurement of velocity and stress fields in complex polymer flows. in 'Proceedings of the XI International Congress on Rheology, Brussels, Belgium' 16-23 (1992). 